\title{
Impacts des pressions climatiques et non climatiques sur les communautés piscicoles de grands fleuves français
}

\author{
Impacts of climatic and non-climatic pressures \\ on fish communities in large French rivers
}

\author{
M. Daufresne \\ Cemagref, UR HYAX, Équipe Écosystèmes Lacustres, 3275 route de Cézanne, CS 40061 , \\ 13182 Aix-en-Provence Cedex 5, France \\ e-mail : martin.daufresne@cemagref.fr
}

\begin{abstract}
Résumé - L'impact écologique du changement climatique n'est plus à démontrer. Cependant, peu d'études ont mis en évidence des règles générales d'influence du réchauffement sur la structure et la diversité des communautés animales, probablement à cause du manque de suivis multi-sites et multi-taxons. L'influence relative des perturbations anthropiques non climatiques est en particulier largement inconnue. Nous présentons ici une méta-analyse portant sur l'effet du changement climatique sur les organismes aquatiques. Les communautés piscicoles de grands fleuves français, soumis à diverses pressions anthropiques, montrent des accroissements significatifs des proportions de poissons méridionaux et thermophiles et des richesses spécifiques au cours des 15 à 25 dernières années. Inversement, l'équitabilité a diminué, soulignant une domination graduelle des peuplements par un nombre réduit d'espèces. Finalement, les abondances globales augmentent, couplées à un rajeunissement et un changement des structures en taille des communautés. Ces changements sont peu influencés par les pressions non-climatiques. Cependant les barrières naturelles ou anthropiques à la migration semblent réduire l'accroissement des proportions de poissons méridionaux. De plus, s'ils ne jouent pas sur les dérives temporelles globales, les centres nucléaires modifient la structure des communautés aval. Cette synthèse de travaux réalisés par le Cemagref pour Électricité de France souligne l'importance des effets du changement climatique sur les communautés animales, même dans des sites perturbés par des pressions non-climatiques.
\end{abstract}

Mots clés - changement climatique, poisson, perturbations anthropiques, communautés, méta-analyse

\begin{abstract}
It is widely accepted that climate change constrains biota. Yet, because of the lack of consistent multi-site and multi-taxon surveys, few studies have addressed general rules about how climate change impacts on structure and diversity of animal communities. Especially, the relative influence of non-climatic anthropogenic disturbances on this impact is fairly unknown. Here, we present a meta-analysis assessing the effect of global warming on stream organisms. Fish communities of large rivers in France undergoing various anthropogenic pressures showed significant increase in proportions of southern and
\end{abstract}


warm-water species and of specific richness during the last 15 to 25 years. Conversely, the equitability decreased, indicating a gradual decrease of the number of dominant species. Finally, the total abundance increased, coupled with rejuvenation and changes in sizestructure of the communities. Most of these effects were not depressed by the strength of non-climatic anthropogenic disturbances. However, natural or anthropogenic barriers to migration seemed to hinder the increase in the proportion of southern species. In addition, if nuclear power plants do not influence global temporal changes, they modify downstream community structures. This synthesis of several works done by the Cemagref for Électricité de France provides new evidence that climate change has deep impacts on communities, even in disturbed sites.

Key words - climate change, fish, anthropogenic disturbances, community, meta-analysis

\section{INTRODUCTION}

Depuis la fin des années 80 , un nombre croissant de travaux dédiés à l'étude des phénomènes climatiques a permis de développer et d'imposer l'hypothèse d'un réchauffement global de la planète. Ainsi, au cours des 140 dernières années, marquées par une forte activité industrielle et des rejets massifs de gaz à effet de serre, la température du globe a progressivement augmenté d'environ $0,6^{\circ} \mathrm{C}$ (IPCC, 2001).

Ce changement climatique confirme l'influence majeure des activités humaines sur l'écosystème terrestre. Son universalité ainsi que sa rapidité le rendent incontournable en écologie. II est en effet essentiel de connaître ses effets précis sur les organismes, d'abord pour en estimer les conséquences, ensuite pour être capable d'en éliminer l'éventuel effet lors de l'étude d'autres processus écologiques. De même, d'un point de vue appliqué, il est primordial de pouvoir prendre en compte les effets du réchauffement climatique dans une optique de gestion des autres contraintes anthropiques exercées sur le milieu. Cela est particulièrement vrai pour les rejets thermiques à l'aval de Centres nucléaires de production d'électricité (CNPE) comme l'ont démontré les mesures prises au cours de l'épisode caniculaire de l'été 2003.

Les travaux relatifs à l'étude des effets du changement climatique ont porté sur des écosystèmes et des organismes très variés, plantes, insectes, invertébrés terrestres et marins, zooplancton, reptiles, amphibiens, oiseaux ou poissons ... (Hughes, 2000 ; Walther et al., 2002 ; Parmesan \& Yohe, 2003). Ces études ont principalement mis en évidence (1) un avancement des phénomènes biologiques printaniers et (2) des modifications des aires de répartition des organismes vers des altitudes ou des latitudes plus élevées, en accord avec leurs préférences thermiques.

Les organismes poïkilothermes, comme les organismes aquatiques, sont de bons modèles biologiques pour étudier les effets du changement climatique. Un certain nombre d'auteurs ont travaillé sur les milieux marins, mais les études relatives aux organismes d'eau douce sont beaucoup plus rares et elles portent essentiellement sur les milieux lacustres. Les quelques travaux relatifs aux cours d'eau confirment les hypothèses de développement 
local d'organismes méridionaux d'eau chaude aux dépens d'organismes septentrionaux d'eau plus froide (Daufresne et al., 2004). Ces observations portent malheureusement sur un seul site ce qui rend difficile l'analyse de la part relative d'autres facteurs, comme les aménagements hydroélectriques, dans les modifications observées. II est donc aujourd'hui essentiel de réaliser de telles analyses sur plusieurs sites différents.

Tel est l'objectif du présent travail lequel vise à préciser les effets relatifs du changement climatique et d'autres pressions anthropiques non-climatiques (aménagements hydroélectriques, rejets thermiques des CNPE) sur les peuplements piscicoles. A cette fin, nous présentons différents travaux réalisés par le Cemagref pour Électricité de France (EDF) et portant sur l'analyse des chroniques de données recueillies autour de 7 CNPE, Nogent-sur-Seine, Belleville-sur-Loire, Chinon, Bugey, Cruas, Saint-Alban et Tricastin (Daufresne et al., 2004; Daufresne \& Boët, 2007). Un certain nombre des résultats présentés ont déjà été publiés (voir en particulier Daufresne \& Boët, 2007). Cependant, compte tenu des enjeux de gestion liés à ces études, l'écriture d'une synthèse en français incluant des détails et des résultats supplémentaires a semblé opportune. Nous analysons d'abord les tendances d'évolution globale des peuplements piscicoles et vérifions leurs relations avec des variables thermiques. Nous détaillons ensuite les modifications de structure des peuplements pour chacun des sites. Les évolutions observées en amont et en aval des rejets thermiques des CNPE sont systématiquement comparées. Enfin, une étude plus précise de l'influence du CNPE Bugey est présentée.

\section{MATÉRIELS ET MÉTHODES}

\subsection{Les données}

Les sites étudiés sont localisés autour de 7 CNPE, situés sur la Seine (Nogent-sur-Seine), la Loire (Belleville-sur-Loire et Chinon) et le Rhône (Bugey, Saint-Alban, Cruas et Tricastin) (Fig. 1). Chaque site est composé de plusieurs stations reparties en amont et en aval du CNPE, représentant un ensemble de $24 \mathrm{com}$ binaisons site $\times$ stations.

Les sites de Belleville, Bugey, Chinon et Nogent sont situés sur le lit mineur naturel du cours d'eau où s'écoule l'ensemble du débit. En revanche, les sites situés sur le Rhône aval sont canalisés et ne reçoivent qu'une partie du débit : les stations de pêche sont alors localisées le long d'ouvrages hydroélectriques, dans des canaux d'amenée ou dans le Rhône court-circuité.

Tous les sites sont échantillonnés par pêche électrique (en continu ou par échantillonnage ponctuel d'abondance, EPA) de 1 à 4 fois par an (Tab. I). Pour chaque pêche, est calculé par espèce, le nombre de poissons pêchés en 20 minutes (capture par unité d'effort ou CPUE). Ces CPUE sont calculées par souci d'homogénéité avec les études préalablement réalisées sur ces données (Cattanéo et al., 2001; Grenouillet et al., 2001; Daufresne et al., 2004, 2005; Daufresne \& Boët, 2007). L'anguille est écartée de l'étude en raison de son caractère amphihalin. 


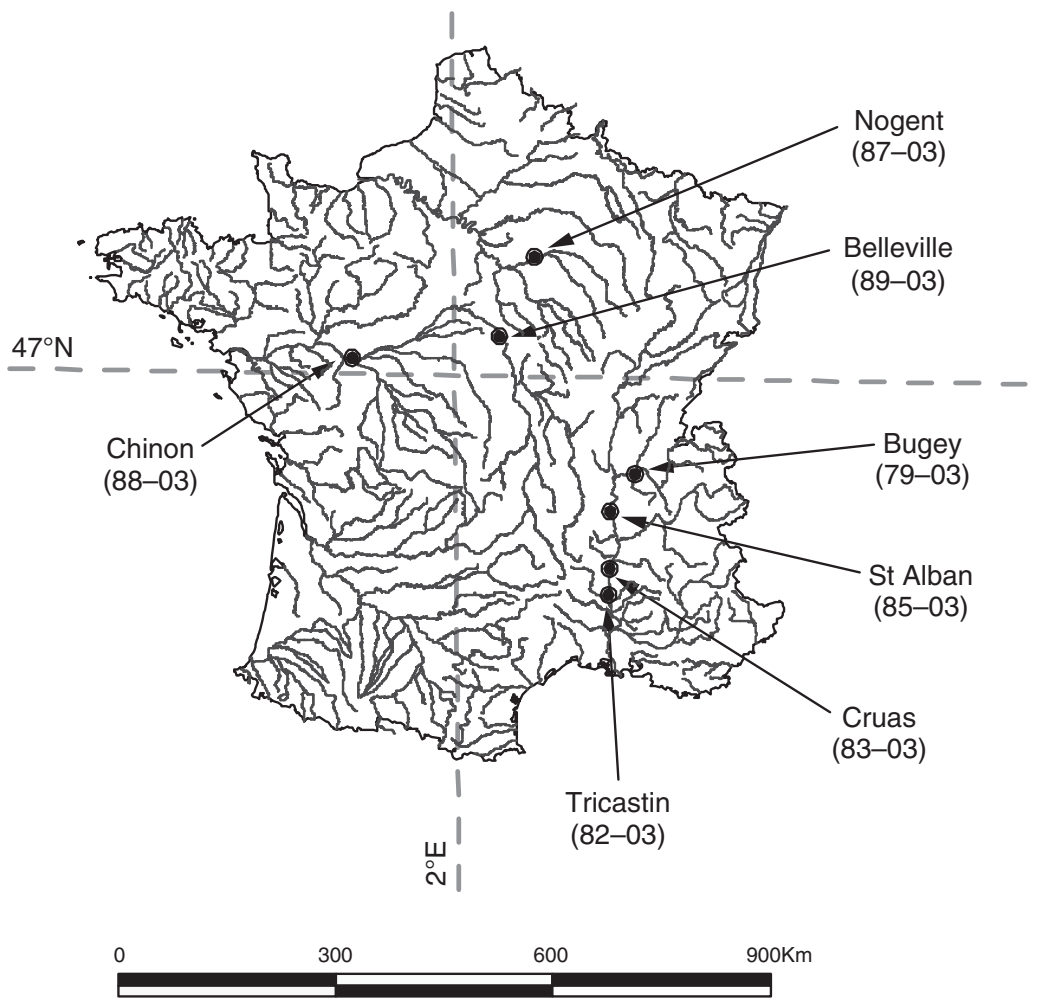

Fig. 1. Localisation des sites d'étude et périodes d'échantillonnage.

Fig. 1. Location of the study areas and sampling periods.

Tableau I. Principales caractéristiques des sites.

Table I. Main properties of the study areas.

\begin{tabular}{lccccc}
\hline & $\begin{array}{c}\text { Superficie } \\
\text { BV }\left(\mathrm{km}^{-2)}\right.\end{array}$ & \multicolumn{2}{c}{ Nb. de stations } & $\begin{array}{c}\text { Effort moyen de pêche } \\
\text { par station (min.) }\end{array}$ & $\begin{array}{c}\text { Nb. de pêches } \\
\text { par an }\end{array}$ \\
\cline { 3 - 4 } & & Amont CNPE & Aval CNPE & & \\
\hline Belleville s/L & 34883 & 1 & 1 & 123,2 & 1 \\
Bugey & 15830 & 3 & 4 & 27,6 & 4 \\
Chinon & 60024 & 1 & 1 & 124,5 & 1 \\
Cruas & 68878 & $1^{*}$ & $2^{*}$ & 25,5 & 4 \\
Nogent s/S & 8939 & 1 & 2 & 57,9 & 1 \\
Saint-Alban & 50736 & 1 & $3^{*}$ & $20(=20 \mathrm{EPA})$ & 4 \\
Tricastin & 74256 & $1^{*}$ & $2^{*}$ & 19,8 & 4 \\
\hline
\end{tabular}

* Sites situé le long d'un ouvrage hydroélectrique (c'est-à-dire $<5 \mathrm{~km}$ de l'ouvrage). 
Pour chaque site, nous disposons également de la température moyenne de l'eau sur la période avril-juin en amont des CNPE. Avril-juin est généralement considéré comme la période de reproduction de la plupart des cyprinidés peuplant les grands cours d'eau français. Plus généralement, cette période printanière est une période clef dans la réponse des organismes au changement climatique. Elle s'est en effet fortement réchauffée dans l'hémisphère nord au cours des 20 dernières années et correspond très souvent à des phases sensibles du cycle de vie des organismes (reproduction, incubation, survie des jeunes de l'année).

\subsection{Méta-analyse (voir Daufresne \& Boët, 2007)}

Nous étudions, dans un premier temps, les tendances d'évolution globale des peuplements piscicoles, c'està-dire tous sites confondus.

Afin de rendre comparables les données des différents sites, nous avons, dans un premier temps, moyenné par an les CPUE par espèce, pour chacun des sites. Nous avons défini une année biologique fondée sur le cycle de vie de la plupart des cyprinidés, du $1^{\mathrm{er}}$ juillet au 30 juin. Les captures réalisées en fin d'année $n$ ou en début d'année $n+1$ sont affectées à la même année biologique. Les sites pouvant être échantillonnés à différentes saisons de l'année, ceci permet de comparer des classes d'âges identiques. Chaque année biologique et pour chaque combinaison site $\times$ station, nous disposons donc finalement d'une CPUE moyenne par espèce.

\subsubsection{Abondance, richesse} spécifique, équitabilité annuelle

Pour chaque année biologique et chaque combinaison site $\times$ station, sont calculés l'abondance totale de poissons (somme des CPUE moyennes), la richesse spécifique (c'est-à-dire le nombre d'espèces dont la CPUE moyenne est non nulle), et l'équitabilité $(E)$ (Begon et al., 1990) avec, pour un ensemble de $i$ espèces :

$$
E=\frac{H}{\ln (R S)}
$$

où

$$
H=-\sum_{i} p_{i} \log \left(p_{i}\right)
$$

$p_{i}$ représentant la probabilité d'occurrence de l'espèce $i$ pour une année biologique donnée et $R S$ étant la richesse spécifique.

\subsubsection{Proportion d'espèces méridionales}

Pour chaque année biologique et chaque combinaison site $\times$ station, nous calculons aussi le pourcentage d'espèces méridionales en termes de CPUE.

À cette fin, pour chaque site, nous travaillons uniquement sur les espèces représentant plus de $95 \%$ des CPUE totales. Pour définir si une espèce $j$ est méridionale dans le site $i$, nous nous basons sur la position relative du site $i$ dans l'aire de répartition de l'espèce $j\left(X_{i, j}\right.$ voir Daufresne et al., 2004). Considérant $L_{j, \text { sup }}, L_{j, \text { inf }}$ et $L_{i}$ comme étant respectivement la latitude nord de l'aire de répartition de l'espèce $j$, la latitude sud de l'aire de répartition de l'espèce $j$ et la latitude 
Tableau II. Latitudes nord et sud des aires de répartition des principales espèces pêchées sur les sites (Bruslé \& Quignard, 2001; Keith \& Allardi, 2001) et latitudes des différents sites d'étude.

Table II. Northern and southern limits of the geographical ranges of the main species sampled in the different study areas (Bruslé \& Quignard, 2001; Keith \& Allardi, 2001) and latitude of these study areas.

\begin{tabular}{lcc}
\hline Espèce & Latitude Sud & Latitude Nord \\
\hline Ablette & 41 & 67 \\
Barbeau & 42 & 57 \\
Bouvière & 36,5 & 61 \\
Brème bordelière & 39 & 65 \\
Brème & 39 & 69 \\
Brochet & 36,5 & 70 \\
Chevaine & 36,5 & 63,5 \\
Gardon & 41 & 66 \\
Goujon & 36 & 67 \\
Hotu & 42,5 & 63 \\
Poisson chat & 37,5 & 63 \\
Perche & 40 & 69,5 \\
Perche soleil & 42 & 61 \\
Rotengle & 36 & 65 \\
Silure & 36 & 60,5 \\
Spirlin & 36 & 62 \\
Tanche & 36 & 64 \\
Vandoise & 41,5 & 70 \\
\hline
\end{tabular}

\begin{tabular}{lc}
\hline Site & Latitude \\
\hline Belleville & 47,5 \\
Bugey & 45,8 \\
Chinon & 47,2 \\
Cruas & 44,6 \\
Nogent & 48,5 \\
Saint-Alban & 44,3 \\
Tricastin & 45,4 \\
\hline
\end{tabular}

du site $i$ (Tab. II), $X_{i, j}$ est calculé comme le rapport :

$$
X_{i, j}=\frac{L_{i}-L_{j, \text { inf }}}{L_{j, \text { sup }}-L_{i}} .
$$

Pour chaque site $i$, le quantile $75 \%$ des $X_{i, j}$ est ensuite calculé et les espèces dont $X_{i, j}$ est supérieur à ce quantile sont considérées comme méridionales. En effet, pour ces espèces, la limite sud de leur aire de répartition est relativement plus éloignée du site $i$ que pour les autres espèces (Fig. 2). Les autres espèces peuvent être considérées comme septentrionales ou « medium », caractéristiques qui seront utilisées dans les analyses ultérieures (analyses par site).
II convient de souligner que le trait latitudinal d'une même espèce varie selon les différents sites.

\subsubsection{Proportion d'espèces thermophiles}

Pour chaque année biologique et chaque combinaison site $\times$ station, nous calculons le pourcentage d'espèces thermophiles, en termes de CPUE. Pour chaque site, nous travaillons uniquement sur les espèces représentant plus de $95 \%$ des CPUE totales. Nous avons défini un premier groupe de six espèces ayant la plus forte affinité pour les fortes températures : la tanche (Tinca tinca), la brème (Abramis brama), la brème bordelière 
(a)

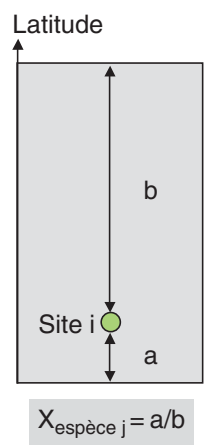

Latitude

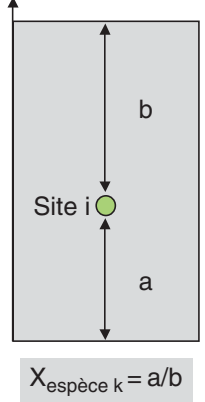

Latitude

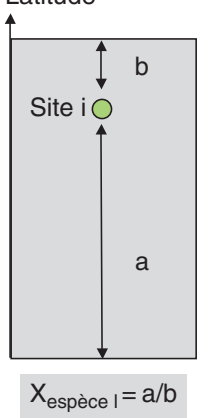

(b)
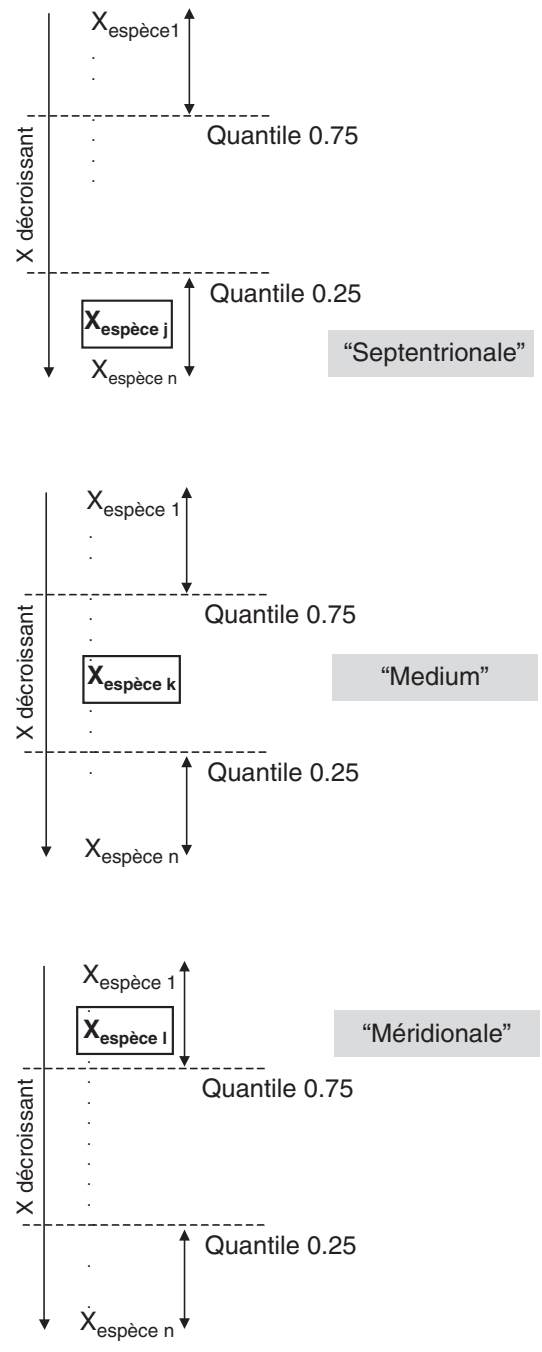

Fig. 2. Position relative du site $i$ dans l'aire de répartition de $n$ espèces et codage du trait latitudinal. (a) Pour chaque espèce, la position relative $(X)$ est d'abord calculée. (b) Les espèces dont le $X$ est inférieur au quantile $25 \%$ sont considérées comme septentrionales; celles dont le $X$ est supérieur au quantile $75 \%$ sont considérées comme méridionales; les autres sont considérées « medium ».

Fig. 2. Relative position $(X)$ of a study area $i$ in the geographical range of species and assignment of latitudinal affinities. (a) For each species $j, X$ is calculated. (b) Species for which $X$ is lower than the $25 \%$ percentile of the $X$ values are considered as "septentrionales"; those having a $X$ value higher than the $75 \%$ percentile of the $X$ values are considered as "meridionales"; others are considered as "medium". 
(Blicca bjoerkna), le rotengle (Scardinius erythrophthalmus), le poisson chat (Ameiurus melas) et le silure (Silurus glanis). Nous avons suivi la classification de Philippart \& Vranken (1983) pour inclure dans ce groupe la tanche, la brème et le gardon (température létale $>34^{\circ} \mathrm{C}$; température de croissance optimale : $20-28^{\circ} \mathrm{C}$; température de reproduction : $20^{\circ} \mathrm{C}$ ). La brème bordelière a été ajoutée seIon Küttel et al. (2002) (température maximale pour les larves, juvéniles et adultes : 28,34 et $36^{\circ} \mathrm{C}$ respectivement; température maximum de reproduction : $23^{\circ} \mathrm{C}$ ), de même que le poisson chat (température maximale pour les adultes : $38^{\circ} \mathrm{C}$; température maximale de reproduction : $20^{\circ} \mathrm{C}$ ). Le silure a été ajouté car cette espèce présente une bonne croissance à forte température $\left(>20^{\circ} \mathrm{C}\right.$; Proteau et al., 1996) et se reproduit à plus de $20^{\circ} \mathrm{C}$ (Bruslé \& Quignard, 2001).

Selon Philippart \& Vranken (1983), nous avons ensuite défini un groupe d'espèces ayant une affinité moyenne avec les fortes températures (température létale $28-34^{\circ} \mathrm{C}$; température de croissance optimale : $14-23^{\circ} \mathrm{C}$; température de reproduction : $\left.7-15^{\circ} \mathrm{C}\right)$ : l'ablette (Alburnus alburnus), le spirlin (Alburnoïdes bipunctatus), le barbeau (Barbus barbus), le chevaine (Leuciscus cephalus), le gardon (Rutilus rutilus) et la bouvière (Rhodeus sericeus). Le barbeau et le spirlin sont considérés comme légèrement sténothermes d'eau froide par Philippart \& Vranken (1983). Toutefois, nous avons inclus le spirlin dans ce second groupe dans la mesure où il croît à de fortes températures (optimum pour les adultes : $18-27^{\circ} \mathrm{C}$; Küttel et al., 2002) et peut se reproduire dans des eaux chaudes (optimum : $12-24^{\circ} \mathrm{C}$; Küttel et al., 2002). De même, le barbeau présente des affinités pour les fortes températures (Kraiem, 1979; Bruslé \& Quignard, 2001) et peut se reproduire jusqu'à $29^{\circ} \mathrm{C}$ (Küttel et al., 2002).

Enfin, nous avons défini un groupe de six espèces avec les plus faibles affinités pour les fortes températures : le brochet (Esox lucius), la perche (Perca fluviatilis), la perche soleil (Lepomis gibbosus), le goujon (Gobio gobio), le hotu (Chondrostoma nasus) et la vandoise (Leuciscus leuciscus). Selon Philippart \& Vranken (1983) le brochet, le hotu et la vandoise ont les mêmes préférences que les espèces du second groupe mais sont définis comme légèrement sténothermes d'eau froide. En effet la reproduction et la croissance des larves de brochet sont optimales à faible température (optimums : $5-10$ et $8-15^{\circ} \mathrm{C}$ respectivement; Küttel et al., 2002), de même que celles de la perche (optimums : $6-15$ et $12-18^{\circ} \mathrm{C}$ respectivement; Küttel et al., 2002). De plus les œufs de perche sont sensibles aux fortes températures (température létale : $16^{\circ} \mathrm{C}$; Elliott, 1981). Les larves de hotu et de vandoise présentent également des préférences pour les faibles températures (température létales : 19 et $18^{\circ} \mathrm{C}$ respectivement; Küttel et al., 2002) et les deux espèces se reproduisent en eau froide (maximum : 16 et $14^{\circ} \mathrm{C}$ pour le hotu et la vandoise respectivement; Küttel et al., 2002). Le goujon a été intégré à ce groupe du fait d'une température de reproduction relativement basse (optimum : $12-17^{\circ} \mathrm{C}$; Küttel et al., 2002) et plus faible que celles du second groupe. Finalement, en dépit de la capacité des adultes de perche 
soleil à supporter de fortes températures (température létale : $35^{\circ} \mathrm{C}$; Küttel et al., 2002), cette espèce a été placée de manière conservative dans ce groupe du fait de sa température optimale de croissance (environ $20^{\circ} \mathrm{C}$; Scott \& Crossman, 1973) et de la sensibilité des jeunes aux fortes températures (température létale : $25^{\circ} \mathrm{C}$; Küttel et al., 2002, fortes mortalités à haute température d'après Dembski et al., 2006).

L'hétérogénéité des données disponibles concernant les préférences thermiques n'a pas permis un codage mathématique des affinités. De ce fait nous avons considéré arbitrairement des affinités pour les fortes températures de $0,75,0,5$ et 0,25 pour les différents groupes. Pour chaque combinaison site $\times$ station, la chronique de CPUE des espèces thermophiles est obtenue en multipliant la matrice des chroniques des CPUE des différentes espèces par leurs préférences en terme de thermophilie (troisième colonne du Tab. III). Finalement la chronique de la proportion d'espèces thermophiles est obtenue en divisant les CPUE des espèces thermophiles par la CPUE totale.

\subsubsection{Méthodes statistiques}

- Tendances long terme

Pour chacune des variables, et pour chaque combinaison site $\times$ station, la tendance est évaluée par la statistique $S$ de Mann-Kendall. Sa variance $V^{*}(S)$ est calculée en prenant en compte les autocorrélations temporelles des séries (Hamed \& Rao, 1998).

Tableau III. Codage du trait d'affinité thermique des principales espèces pêchées sur les sites. Table III. Affinities for high temperature of the main species sampled in the study areas.

\begin{tabular}{lcc}
\hline Espèce & Sténotherme d'eau froide & Thermophile \\
\hline Ablette & 0,5 & 0,5 \\
Barbeau & 0,5 & 0,5 \\
Bouvière & 0,5 & 0,5 \\
Brème bordelière & 0,25 & 0,5 \\
Brème & 0,25 & 0,75 \\
Brochet & 0,75 & 0,25 \\
Chevaine & 0,5 & 0,5 \\
Gardon & 0,5 & 0,5 \\
Goujon & 0,75 & 0,25 \\
Hotu & 0,75 & 0,25 \\
Poisson chat & 0,25 & 0,75 \\
Perche & 0,75 & 0,25 \\
Perche soleil & 0,75 & 0,25 \\
Rotengle & 0,25 & 0,75 \\
Silure & 0,25 & 0,75 \\
Spirlin & 0,5 & 0,5 \\
Tanche & 0,25 & 0,75 \\
Vandoise & 0,75 & 0,25 \\
\hline
\end{tabular}


Pour une série d'observations $\times=$ $x_{1}, x_{2}, \ldots, x_{n}, S$ est calculée selon :

$$
\begin{aligned}
& S=\sum_{i<j} \operatorname{sgn}\left(x_{j}-x_{i}\right) \\
& \text { où } \\
& \operatorname{sgn}\left(x_{j}-x_{i}\right)=\left\{\begin{array}{c}
1 \text { si } x_{i}<x_{j} \\
0 \text { si } x_{i}=x_{j} \\
-1 \text { si } x_{i}>x_{j}
\end{array}\right.
\end{aligned}
$$

D’après Kendall (1955), la variance de $S$, var(S), est donnée par :

$$
\operatorname{var}(S)=\frac{n(n-1)(2 n+5)}{18}
$$

Pour certains types de séries temporelles $(\mathrm{AR}(1)$ et $\mathrm{MA}(1))$, Hamed \& Rao (1998) ont montré théoriquement que les autocorrélations positives tendent à augmenter la variance de $S$. Dans ce sens, la variance de $S$ va être sous estimée par var(S). On observera l'effet inverse si les autocorrélations sont négatives. Les auteurs proposent donc d'appliquer une correction à $\operatorname{var}(S)$ en prenant en compte les autocorrélations temporelles significatives. Pour ce faire, une estimation non paramétrique de la tendance (décrite par Sen, 1968) est soustraite de la série $X$ et les autocorrélations entre les rangs de cette nouvelle série sont calculées. Les coefficients d'autocorrélation significativement différents de zéro $(\rho s(i)$ au pas de temps $i)$ sont alors utilisés pour calculer la variance corrigée de $S, V^{*}(S)$ :

$$
V^{*}(S)=\operatorname{var}(S) \text { Cor }
$$

où Cor est la correction due aux autocorrélations

$$
\begin{aligned}
\text { Cor }= & 1+\frac{2}{n(n-1)(n-2)} \\
& \times \sum_{i=1}^{n-1}(n-i)(n-i-1) \\
& \times(n-i-2) \rho_{S}(i)
\end{aligned}
$$

- Influence de la température à l'échelle inter-annuelle

Pour chacune des variables et pour chaque combinaison site $\times$ station, l'effet de la température moyenne de reproduction a été évalué en considérant la pente $b$ de la régression linéaire entre les deux variables. De même que pour les statistiques de tendances, les autocorrélations des séries ont été prises en compte pour corriger la variance de $b$. Nous avons pour cela calculé un nombre corrigé d'observations $\left(n^{*}\right)$ en suivant la méthodologie décrite par Pyper \& Petermann (1998). Pour des séries de longueur $n$, les auteurs recommandent d'estimer les $n / 5$ premiers coefficients d'autocorrélation $\left(r_{x x}(j)\right)$ (c'est-à-dire aux pas de temps $1,2, \ldots n / 5)$ des deux séries selon la méthode de Chatfield (1989, p. 50) :

$$
r_{x x}(j)=\frac{n}{n-j} \frac{\sum_{t=1}^{n-j}\left(x_{t}-\bar{x}\right)\left(x_{t+j}-\bar{x}\right)}{\sum_{t=1}^{n}\left(x_{t}-\bar{x}\right)^{2}} .
$$

Le nombre corrigé d'observations $n^{*}$ est alors évalué par :

$$
\frac{1}{n^{*}} \approx \frac{1}{n}+\frac{2}{n} \sum_{j=1}^{n / 5} \mathrm{r}_{\mathrm{XX}}(j) \mathrm{r}_{\mathrm{YY}}(j) .
$$


- Méta-analyse

Pour évaluer globalement, c'est-à-dire tous sites confondus, les tendances long terme et l'effet de la température à l'échelle inter-annuelle, nous avons effectué une méta-analyse pondérée par la variance en utilisant les statistiques $S$ et $b$ comme « effect sizes » (Gurevitch \& Hedges, 1993). Pour chaque variable, on a tout d'abord évalué les valeurs moyennes globales de $S$ et $b$ et leurs intervalles de confiance à $95 \%$ par bootstrap. Pour évaluer l'influence des contraintes anthropiques non-climatiques (CNPE et aménagement hydroélectrique) nous avons ensuite défini deux variables de catégories. La première discrimine les stations situées en amont vs. aval des CNPE. La deuxième discrimine les stations situées ou non le long d'ouvrages hydroélectriques (Tab. I). Nous avons utilisé un modèle mixte pour tester l'influence de ces variables sur l'hétérogénéité des réponses en divisant l'hétérogénéité totale en hétérogénéité intra- et inter-groupe $\left(Q_{b}\right)$. La significativité des $Q b$ a été évaluée par 1000 permutations aléatoires et des corrections de Bonferroni (Sokal \& Rohlf, 1995). Dans la mesure où ces corrections sont connues pour être conservatives, les résultats significatifs à $10 \%$ sont discutés (Hillebrand, 2004).

\subsection{Analyses par site}

Dans un deuxième temps, nous avons étudié les tendances d'évolution des structures des différents peuplements, site par site. Pour chaque site, nous avons travaillé uniquement sur les espèces représentant plus de $95 \%$ des CPUE totales. Dans un premier temps, pour normaliser les données, les CPUE moyennes annuelles ont été transformées en In(CPUE + 1).

\subsubsection{Méthodes statistiques}

Pour chaque site, nous avons réalisé des analyses factorielles des correspondances (AFC) sur les tableaux regroupant les chroniques des In(CPUE + 1) moyennes par espèce. Cela permet de résumer chaque année la structure des communautés par un nombre réduit de coordonnées factorielles plutôt que par l'abondance des différentes espèces du peuplement.

Les modifications de structure des peuplements, révélées par l'analyse des axes factoriels, sont interprétées à la fois en terme d'espéces et en terme de traits écologiques. Nous avons défini deux traits, l'un décrivant les préférences thermiques des espèces, l'autre la position relative des sites dans leurs aires de répartition. Le premier trait comporte 2 modalités codées selon le tableau III. Le deuxième trait, dit latitudinal, comporte 3 modalités (c'est-à-dire « méridionale », « medium » et " septentrionale ») codées en 0-1 (cf. Fig. 2 et Tab. IV).

Les tableaux de traits sont obtenus en multipliant les données In(CPUE+1) par les modalités par trait $\times$ espéces (cf. Tab. III pour la thermie). II s'agit en fait de répartir la CPUE totale non plus entre les différentes espèces mais entre les différents traits.

L'évolution fonctionnelle des communautés est évaluée en projetant les tableaux de traits sur les plans factoriels des analyses multivariées comme individus supplémentaires, c'est-à-dire 
Tableau IV. Exemple de préférence latitudinale des principales espèces pêchées à Bugey. Table IV. Example of latitudinal affinities of the main species sampled at Bugey.

\begin{tabular}{lccc}
\hline & méridionale & medium & septentrionale \\
\hline Ablette & 0 & 1 & 0 \\
Barbeau & 0 & 1 & 0 \\
Chevaine & 1 & 0 & 0 \\
Gardon & 0 & 1 & 0 \\
Goujon & 0 & 1 & 0 \\
Hotu & 0 & 0 & 1 \\
Spirlin & 1 & 0 & 0 \\
Vandoise & 0 & 0 & 1 \\
\hline
\end{tabular}

qu'ils sont représentés graphiquement sans contribuer aux analyses.

Afin d'appréhender l'effet des CNPE sur les communautés, nous avons comparé les chroniques de coordonnées factorielles des stations situées ou non dans les veines d'eau réchauffées. En dehors de ces aspects temporels, et comme pour la métaanalyse, nous avons également appréhendé l'effet des CNPE sur les structures globales des communautés dans les sites soumis ou non à l'influence des veines d'eau réchauffées. Pour ce faire, nous avons évalué la significativité des variances inter-groupe (soumis vs. non soumis à la veine chaude) par des tests de permutations (1000 permutations).

\subsubsection{Cas particulier de Bugey}

Dans la mesure où le changement climatique et les CNPE peuvent avoir des effets sur des espèces plus marginales du peuplement, une AFC a également été réalisée sur les chroniques des $\ln (\mathrm{CPUE}+1)$ de toutes les espèces présentes dans plus de $5 \%$ des pêches à Bugey. Ce site a été choisi dans la mesure où il présente les chroniques les plus longues et le plus grand nombre de stations. Ce type d'analyse permet d'appréhender l'effet du réchauffement et du CNPE sur l'ensemble des espèces, y compris les espèces faiblement représentées. En revanche, du fait du grand nombre d'espèces considérées et du manque de connaissances des préférences thermiques des espèces européennes, une analyse fonctionnelle n'a pas pu être envisagée.

Toutes les analyses ont été réalisées en utilisant le logiciel $R$ ( $R$ Development Core Team, 2008).

\section{RÉSULTATS}

Au cours des périodes d'étude, la température moyenne de la période de reproduction (avril-juin) en amont des sites d'étude a eu tendance à augmenter (test de Mann-Kendall modifié, $P<0,01, n=25$, Fig. 3). Nous observons un bon synchronisme des chroniques d'anomalies thermiques à large échelle spatiale et les températures d'eau sont significativement corrélées (tests modifiés de Pearson (Pyper \& 


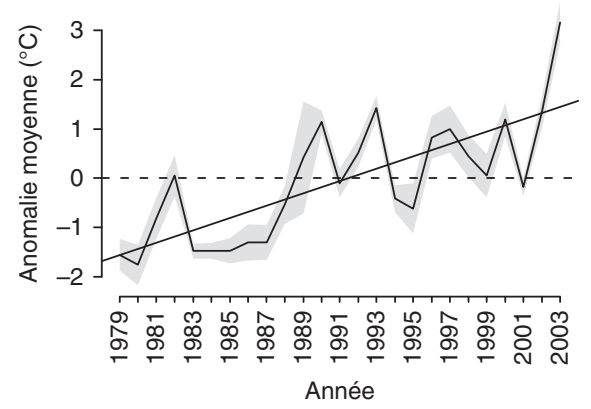

Fig. 3. Anomalie moyenne annuelle ( \pm intervalle de confiance à $95 \%$ ) des températures de la période de reproduction (avril-juin) en amont des sites d'étude. La tendance est figurée $(Y(t)=$ $0,12 t-1,56)$.

Fig. 3. Annual mean anomalies ( $\pm 95 \%$ confidence intervals) of temperature during the reproduction period (April-June). Temperature was recorded upstream from the study areas. The trend is shown $(Y(t)=0.12 t-1.56)$.

Peterman, 1998), $P<0,01$ ) aux températures maximales annuelles mesurées à proximité des sites $(60,36,170$, 8, 14, 83 et $26 \mathrm{~km}$ de Belleville, Bugey, Chinon, Cruas, Nogent, St Alban et Tricastin respectivement; données fournies par Météo-France). Cela suggère que les augmentations de températures de l'eau sont largement imputables au changement climatique.

\subsection{Méta-analyse (voir Daufresne \& Boët, 2007)}

Nous observons des augmentations significatives des abondances totales et des proportions d'espèces thermophiles sur l'ensemble des sites et une diminution significative de l'équitabilité (Figs. 4a et 5). Des mesures de tailles individuelles à Bugey suggèrent que la décroissance des abondances totales pourrait être couplée à une augmentation importante des proportions de petits individus (Fig. 6). La richesse spécifique augmente également significativement et de manière un peu plus importante dans les sites situés le long d'ouvrages hydroélectriques $\left(Q_{b}=10,70, P<0,1\right.$ après correction de Bonferroni). Finalement, la proportion d'espèces méridionales augmente dans les sites non localisés le long d'ouvrages hydroélectriques $\left(Q_{b}=13,04, P<0,1\right.$ après correction de Bonferroni). À l'échelle interannuelle, l'abondance totale, la richesse spécifique et la proportion d'espèces méridionales sont positivement corrélées à la température de la période de reproduction, tandis que l'équitabilité est négativement corrélée (Fig. 4b). La proportion d'espèces thermophiles est positivement corrélée à la température dans les sites localisés le long d'ouvrages hydroélectriques mais négativement corrélée dans les autres sites $\left(Q_{b}=\right.$ $13,40, P<0,05$ après correction de Bonferroni).

Nous n'avons pas observé d'effets significatifs des CNPE sur les différents phénomènes observés $\left(Q_{b}\right.$ variant de $4,8 \times 10^{-4}$ à 0,9$)$. En dehors des effets temporels, des ANOVAs ont montré un effet globalement négatif des ouvrages hydroélectriques sur l'équitabilité $(P<0,01$ après correction de Bonferroni, Fig. 5c) et sur la proportion d'espèces thermophiles (supérieure dans les sites localisés le long des ouvrages, $P<0,01$ après correction de Bonferroni, Fig. 5d). Aucun autre effet global des ouvrages hydroélectriques et des CNPE n'a pu être mis en évidence (ANOVAs, $P>$ 0,05 après correction de Bonferroni dans tous les cas). 

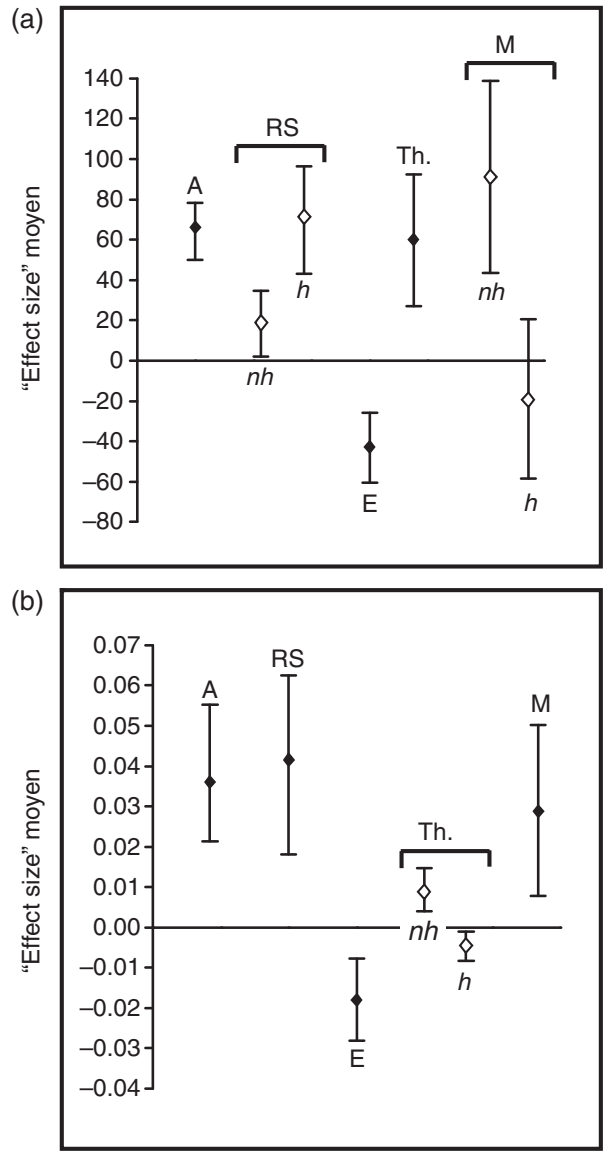

Fig. 4. "Effect sizes " moyens ( \pm intervalle de confiance à $95 \%$ ). (a) Statistique de tendance $S$ de l'abondance totale $(A)$, la richesse spécifique (RS), l'équitabilité $(E)$, les proportions d'espèces thermophiles (Th.) et méridionales (M). Les différences significatives de moyennes entre les sites localisés le long d'ouvrages hydroélectriques $(h)$ ou non $(n h)$ sont représentées. (b) Pente $b$ des relations entre $A, R S, E$, Th. et $M$ et la température de période de reproduction. Les différences significatives de moyennes entre les sites localisés le long d'ouvrages hydroélectriques $(h)$ ou non $(n h)$ sont représentées. Pour accroître la lisibilité, les valeurs de $b$ (et les intervalles de confiance) de $A$ et RS sont divisées par 1000 et 10 respectivement. Le non chevauchement des intervalles de confiance avec 0 indique des effets significatifs au risque de $5 \%$.

Fig. 4. Mean effect sizes ( $\pm 95 \%$ confidence intervals). (a) Mean for the Trend $S$ of the total abundance of fish (A), the specific richness (SR), the Shannon equitability index (E), the proportion of southern species to the total abundance $(\mathrm{M})$ and the proportion of warm-water species to the total abundance (WW) at the different sampling sites. Significantly different means at sampling sites located along hydropower schemes $(h)$ or not $(n h)$ are represented (open diamonds). (b) Mean for the Slope of the linear regression with temperature during the reproduction period $b$ of $A, S R, E, M$ and WW at the different sampling sites. Significantly different means at sampling sites located along hydropower schemes $(h)$ or not $(n h)$ are represented (open diamonds). To increase readability, $b$ values (and confidence intervals) of A and SR were divided by 1,000 and 10 respectively. Non-overlapping of confidence intervals with zero indicates significant effects. 
(a)

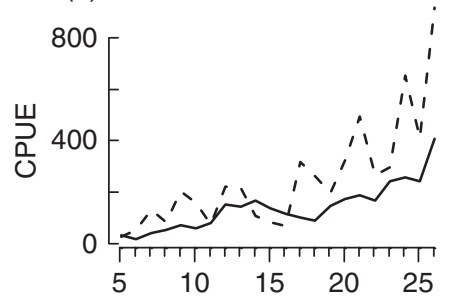

(c)

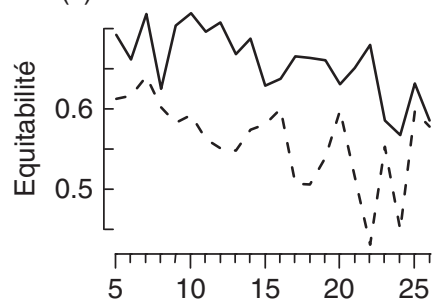

(e)

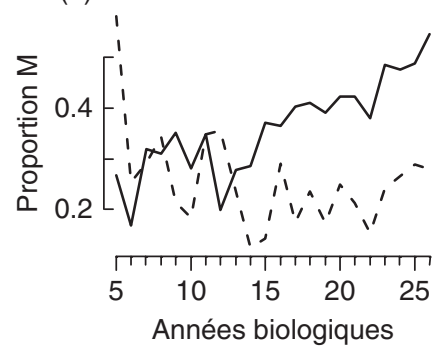

(b)

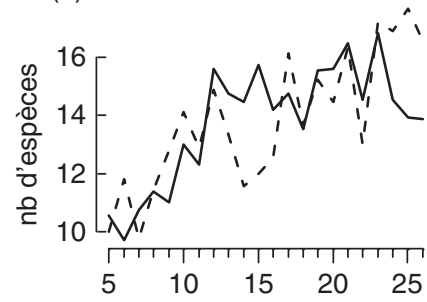

(d)

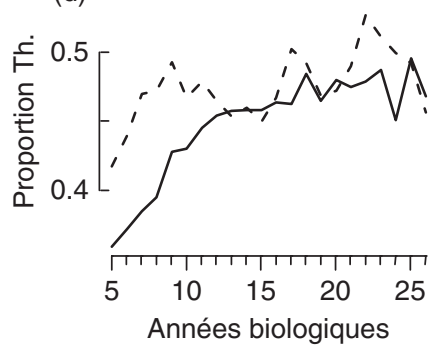

Fig. 5. Chroniques des moyennes annuelles de : (a) l'abondance totale, (b) la richesse spécifique, (c) l'équitabilité et des proportions (d) d'espèces thermophiles et (e) méridionales dans les stations situées le long d'ouvrages hydroélectriques (lignes pointillées) ou non (lignes continues).

Fig. 5. Temporal changes (annual means) in total abundance, community structure and diversity at the sampling sites located along hydropower schemes (dotted lines) or not (continuous lines). (a) Total abundance (expresses as CPUE). (b) Specific richness (expresses as number of species). (c) Shannon equitability index. (d) Proportion of warm-water species to the total abundance. (e) Proportion of southern species to the total abundance.

\subsection{Analyses par site}

Tous les sites présentent des tendances de modification de la structure de leur peuplement dans le temps (Fig. 7). D'un point de vue fonctionnel, on retrouve en partie les différences observées dans l'analyse globale. Les tendances en termes de préférences latitudinales, avec un développement des espèces méridionales, se retrouvent bien pour la plupart des sites sauf Cruas et Saint-Alban. De manière générale, elles s'avèrent moins 


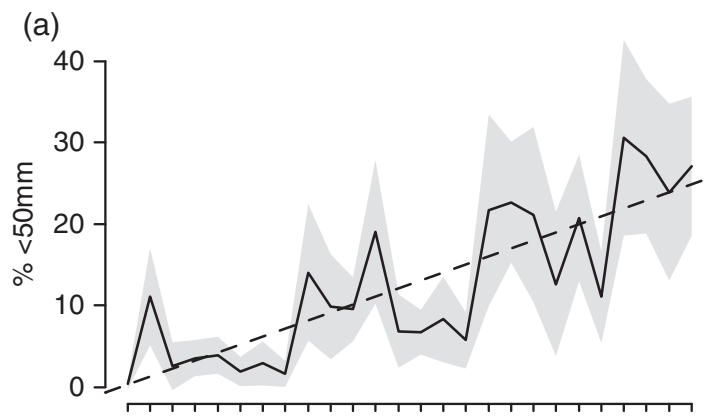

(b)

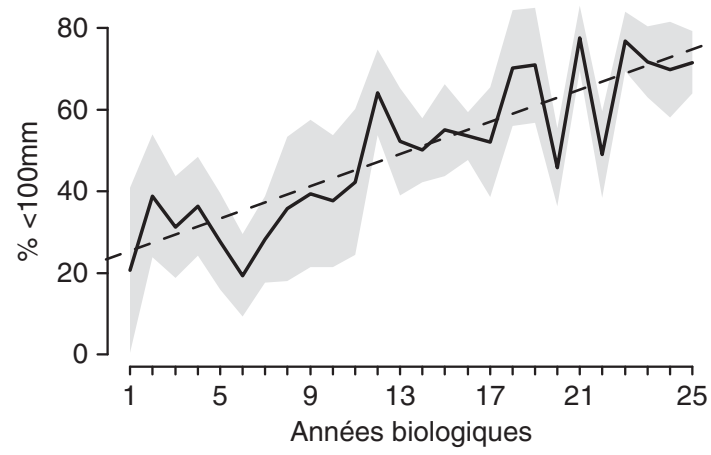

Fig. 6. Pourcentages annuels moyens ( \pm intervalle de confiance à $95 \%$ ) de poissons de petite taille pêchés à Bugey. (a) Poissons inférieurs à $50 \mathrm{~mm}$. (b) Poissons inférieurs à $100 \mathrm{~mm}$. Les tendances sont figurées $(Y(t)=0,98 t-0,66, Y(t)=2,00 t-22,49$ pour les poissons inférieurs à 50 et $100 \mathrm{~mm}$ respectivement).

Fig. 6. Annual mean percentages ( $\pm 95 \%$ confidence intervals) of small fish sampled at Bugey. (a) Fish smaller than $50 \mathrm{~mm}$. (b) Fish smaller than $100 \mathrm{~mm}$. The trend are shown $(\mathrm{Y}(\mathrm{t})=0.98 \mathrm{t}-$ 0.66 for fish smaller than $50 \mathrm{~mm}$ and $Y(t)=2.00 \mathrm{t}-22.49$ for fish smaller than $100 \mathrm{~mm}$ ).

marquées pour les sites du Bas Rhône (Cruas, Saint-Alban et Tricastin), soumis à l'influence des aménagements hydroélectriques. Concernant les préférences thermiques, seul Bugey présente un développement marqué d'espèces thermophiles. Cela signifie que les évolutions relatives aux préférences thermiques mises en évidence par la méta-analyse ne sont pas les patrons majeurs (en termes de variabilité globale) de structuration des communautés.
La variabilité inter-annuelle représente une part importante (variant de 30 à $78 \%$ ) de la variabilité totale de ces jeux de données. En comparaison, la variabilité inter-CNPE (c'està-dire entre les stations situées ou non dans les rejets thermiques des CNPE) est faible (variant de 1 à $18 \%$ de la variabilité totale). Dans ce sens, les modifications temporelles des structures des communautés sont très similaires dans les différents sites (Fig. 5). En dehors de ces aspects 

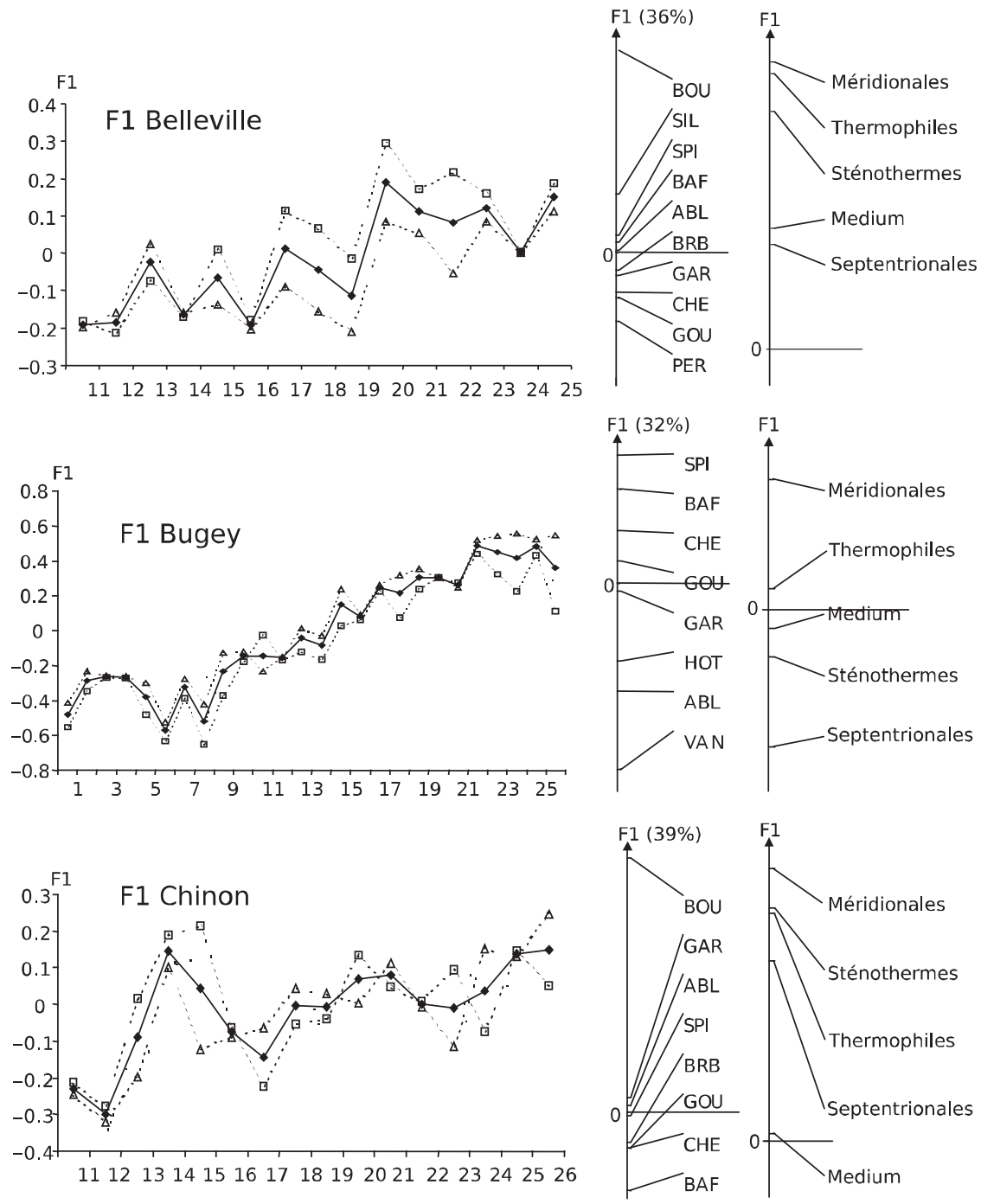

Années Biologiques

Fig. 7. Chroniques des coordonnées factorielles des échantillons (moyennées pour toutes les stations) et contribution des espèces sur les axes des AFC réalisées sur les données des différents sites. Seuls les premiers axes présentant des tendances temporelles significatives sont représentés. L'interprétation des axes en termes de traits écologiques est donnée. Les évolutions sont également représentées pour les stations situées en amont des CNPE (carrés ouverts) et en aval des CNPE (triangles ouverts).

Fig. 7. Annual mean scores (all sampling sites pooled) of samples and species scores on COA axes for the different study areas. For each study area, only the first axis with a significant temporal trend is shown. Temporal trends in sampling sites located upstream (open squares) and downstream (open triangles) from the nuclear power plant are represented. 

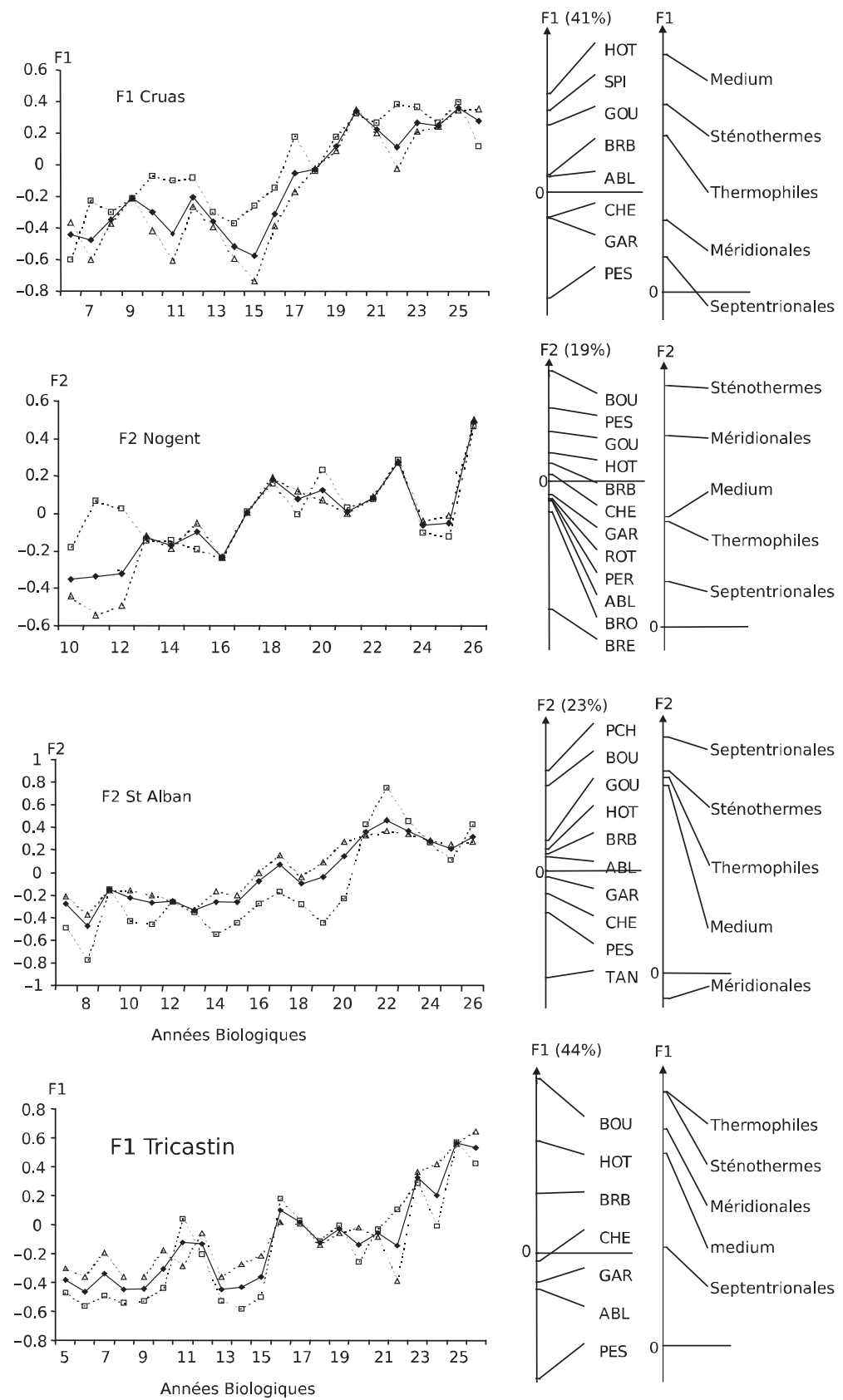

Fig. 7. Suite.

Fig. 7. Continued. 
temporels, il convient cependant de noter qu'un effet des CNPE (amont vs. aval) sur les structures globales des communautés a été observé pour les sites de Belleville, Bugey, Cruas et Saint-Alban $(P<0,01$ après correction de Bonferroni dans tous les cas). Cela ce traduit par exemple par des abondances de perche plus importantes et de bouvière moins importantes dans la station située en aval du CNPE Belleville ou par des abondances de spirlin plus importantes et de vandoise moins importantes dans les stations situées dans le panache thermique du CNPE Bugey (Fig. 7).

\subsubsection{Cas particulier de Bugey}

La variance inter-CNPE $(P<0,01)$ représente seulement $1,2 \%$ de la variance totale du tableau type AFC (contre $17,6 \%$ pour l'interannuelle) lorsque les espèces marginales sont prises en compte. Le tableau apparaît peu structuré (Fig. 8) et de ce fait il est évident qu'il convient d'interpréter les résultats avec prudence. Les trois premiers axes n'expliquent que $28,5 \%$ de la variance totale $(11,6 \%$, $9,2 \%$ et $7,6 \%$ pour les axes F1, F2 et F3 respectivement). Cependant, il est intéressant de noter que les deux premiers axes discriminent bien les stations « réchauffées » par l'effluent du CNPE et les stations « non réchauffées », en particulier l'axe F2. Cela traduit principalement des proportions plus importantes de loches, épinoches et de truites dans les stations « non réchauffées » et des proportions plus importantes de brèmes bordelières, blageons et de silures dans les stations « réchauffées » (Fig. 9).

\section{DISCUSSION}

\subsection{Modifications long termes des structures de communautés (voir Daufresne \& Boët, 2007)}

L'augmentation de la température de l'eau sous la contrainte du changement climatique s'est accompagnée de profondes modifications des abondances globales de poissons et de la structure et de la diversité des communautés dans les grands fleuves français (Fig. 4). Ces changements, comparables pour des communautés soumises à des types et des niveaux de contraintes non climatiques très diverses, soulignent l'impact du changement climatique sur les communautés piscicoles.

Certains changements sont quantitativement très importants. Par exemple, nous avons observé une augmentation moyenne ( \pm IC à $95 \%$ ) des abondances totales de $+232 \%$ $( \pm 103 \%)$ entre les 5 premières et les 5 dernières années des différents suivis. D'après Daufresne \& Boët (2007), cette modification pourrait s'expliquer par un rajeunissement des populations (avec des proportions de jeunes individus plus importantes) et par un développement global des espèces de petites tailles (ex : le spirlin ou l'ablette). Quoiqu'il en soit, il est intéressant de noter que ces changements de structure en taille sont en accord avec les diverses règles écogéographiques qui stipulent que les tailles moyennes tendent à décroitre avec la latitude (voir Knouft, 2004 pour un exemple sur les communautés piscicoles).

En dehors des tendances sur les abondances, nous avons observé un accroissement de la richesse 


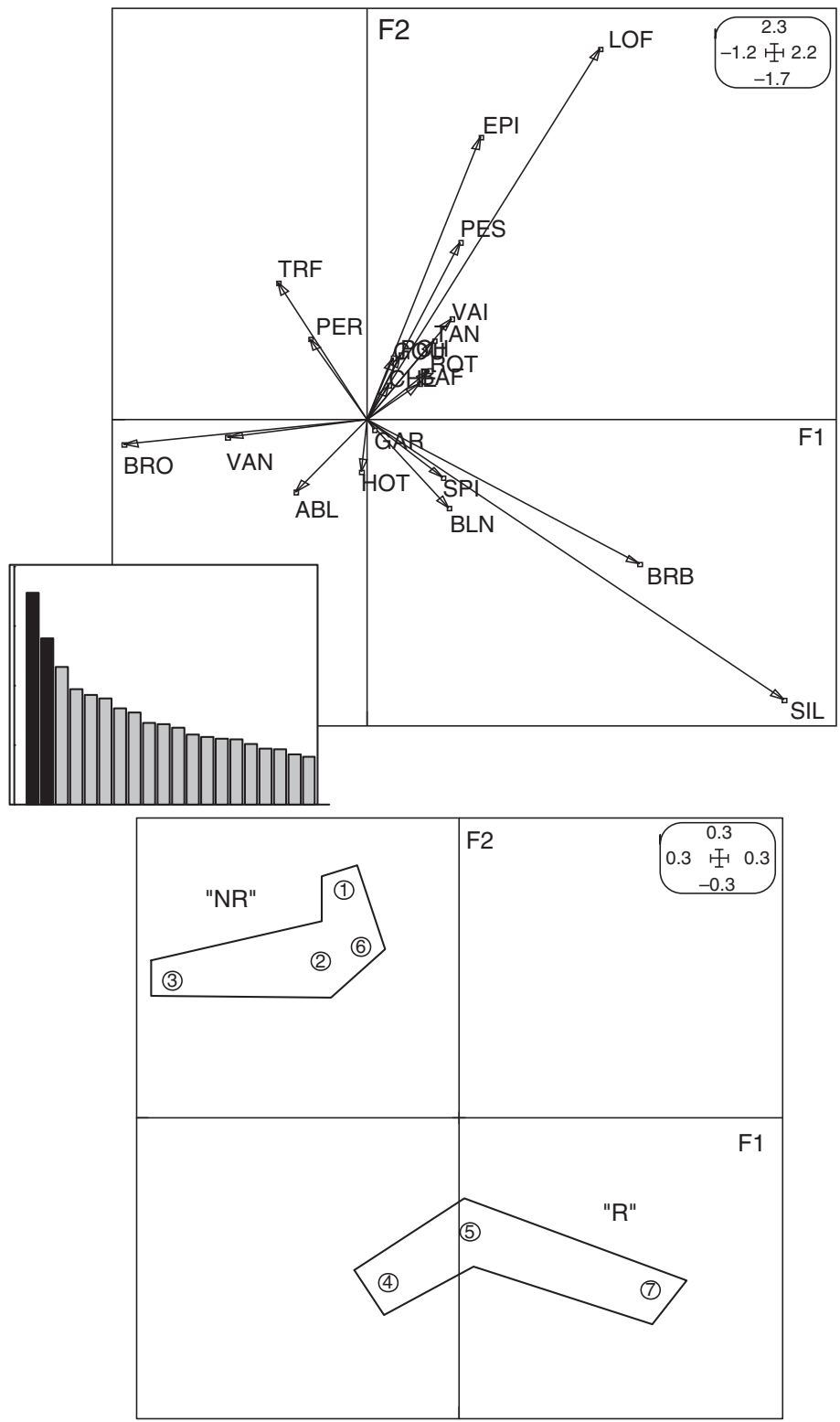

Fig. 8. Coordonnées factorielles des espèces et des stations sur le plan F1-F2 de l'AFC réalisée sur les $\ln (C P U E+1)$ par pêche et par espèces à Bugey. Toutes les espèces présentes dans plus de $5 \%$ des pêches sont considérées (contrairement à la figure 7). "NR " = stations non réchauffées; « $R$ » = stations réchauffées. Les polygones n'ont pas de valeur statistique ou mathématique.

Fig. 8. Scores of the species and the sampling sites on the F1-F2 factorial plan of the COA performed on the In(CPUE +1$)$ by sampled and species at Bugey. All the species involved in the analysis contribute for more than $5 \%$ of the total abundance (conversely to Fig. 7). "NR" = sampling sites nonimpacted by the nuclear power plant, "R" = sampling sites impacted by the nuclear power plant. The polygons have no mathematical significance. 

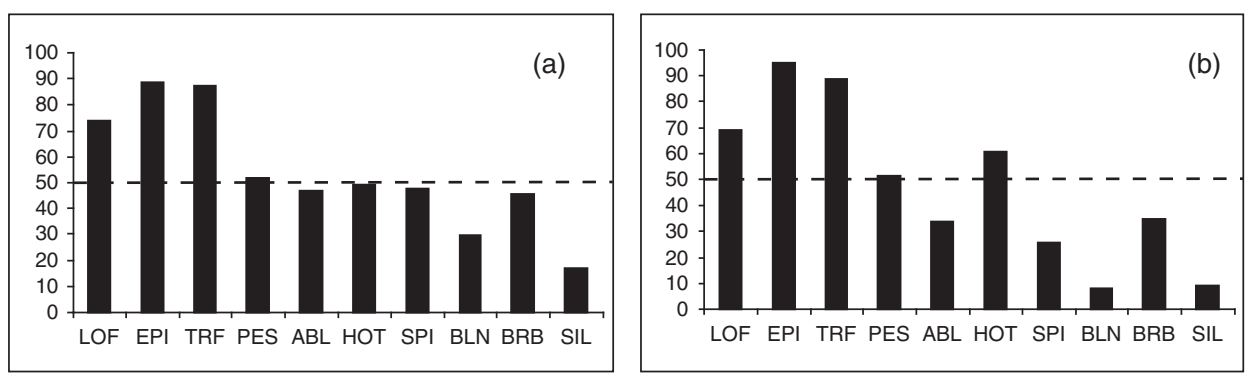

Fig. 9. Pourcentages de l'occurrence (a) et de l'abondance totale (b) de 10 espèces dans les stations "non réchauffées » à Bugey. Notez que, par complémentarité, les pourcentages restant concernent les stations dites " réchauffées".

Fig. 9. Percentages of (a) the total occurrence and (b) the total abundance of 10 species at the sites submitted to the impact of the nuclear power plant at Bugey. Note that the remaining percentages are found at the sites non-impacted by the nuclear power plant.

spécifique et une décroissance de l'équitabilité. D'après Connell (1978) et Wilson (1990), l'accroissement de la richesse spécifique n'est pas surprenant. En effet, un changement graduel des conditions environnementales est l'une des hypothèses les plus plausibles pour expliquer le "paradoxe du plancton » de Hutchinson (1961), c'est-à-dire le fait d'observer un grand nombre d'espèces partageant des ressources similaires (un phénomène a priori contradictoire avec la théorie de l'exclusion compétitive). Cette hypothèse repose principalement sur le postula de la générale non atteinte d'équilibre de structure des communautés. Sous contrainte d'un changement climatique une espèce ne va pas avoir le temps d'exclure les compétiteurs avant d'être elle-même contrainte par le climat. Dans ce sens, les communautés regroupent toujours un mélange d'espèces favorisées par le climat actuel et d'espèces favorisées par le climat passé. Ces dernières ne disparaissent pas nécessairement subitement mais sont éliminées seulement après plusieurs générations. Si elle est explicable, cette augmentation de richesse spécifique ne pourrait cependant être que transitoire dans le cadre du changement climatique actuel. En effet, Connell (1978) souligne que des changements trop rapides ne permettent pas aux communautés d'évoluer et ne vont donc pas promouvoir la biodiversité. Dans ce sens, Daufresne \& Boët (2007) ont montré que les augmentations de richesse sont plus le fait d'une augmentation des occurrences d'espèces déjà présentes que de l'arrivée de nouvelles espèces au sens strict. La baisse de l'équitabilité souligne également un effet négatif du changement climatique sur la biodiversité. Seul un nombre très réduit d'espèces tend à bénéficier du réchauffement.

Finalement, nous avons observé un accroissement des proportions d'espèces thermophiles et un accroissement des espèces méridionales dans les stations non soumises à l'influence d'aménagements hydroélectriques. Ces résultats sont cohérents 
avec les études portant sur l'impact du changement climatique sur les organismes (Parmesan \& Yohe, 2003; Root et al., 2003; Walker et al., 2006). II est intéressant de noter que, localement, les modifications en termes de préférences latitudinales sont conséquentes au regard de la variabilité globale des jeux de données (puisqu'elles sont visibles sur les premiers axes des AFC, Fig. 7). Cela est moins vrai pour les modifications en termes de thermophilie. Le développement d'espèces thermophiles est réel à l'échelle globale (Fig. 4) mais il ne semble pas représenter un phénomène majeur contrôlant la variabilité locale des communautés (Fig. 7). Nous ne pouvons cependant pas exclure l'influence du codage des préférences thermiques sur ces résultats. Les études portant sur ces préférences sont très hétérogènes et ne concernent qu'un nombre restreint d'espèces. Cela permet d'avoir une idée générale des préférences mais qui est (1) finalement peu précise et (2) ne tient pas compte d'une quelconque variabilité spatiale (à l'inverse des préférences latitudinales qui sont codées pour chaque combinaison espèce $\times$ site). Si ces imprécisions ne masquent pas une modification des structures de communautés à l'échelle globale, il est possible qu'elles le fassent à l'échelle locale.

\subsection{Influence des pressions anthropiques non climatiques}

\section{- Aspects temporels}

Nous n'avons pas trouvé d'effets significatifs des CNPE sur les modifications globales d'abondance totale et de structure et diversité des communautés. De même, à l'échelle inter-annuelle, les CNPE n'influencent pas les relations entre la température de reproduction et les caractéristiques des communautés. Cependant, il est important de noter que nous ne disposions pas d'informations quant aux caractéristiques des veines chaudes à l'aval des CNPE. Or, il existe des différences de niveaux de réchauffement entre les CNPE fonctionnant en circuit fermé (ex : Nogent, Cruas) ou en circuit semi ouvert (comme Bugey). Les résultats d'une analyse comparative des structures de communautés en amont vs. aval des CNPE sont donc à prendre avec prudence. La mise en place d'une cartographie précise des panaches thermiques paraît fondamentale pour infirmer ou confirmer les phénomènes mis en évidence.

Les aménagements hydroélectriques ont globalement peu d'effet sur les modifications temporelles des structures des communautés. Ils semblent cependant (1) empêcher l'augmentation des proportions d'espèces méridionales et (2) diminuer le niveau d'augmentation de la richesse spécifique (Fig. 4a). Ce dernier effet, significatif au risque de $10 \%$, est cependant quantitativement peu important (Fig. 5b) et est probablement surtout dû à un accroissement plus graduel de la richesse dans les stations soumises à l'impact des aménagements. Concernant l'effet des aménagements sur les proportions d'espèces méridionales, deux explications peuvent être avancées. II est d'abord vraisemblable que les barrages représentent un frein à la migration d'espèces méridionales vers ces stations. En dehors de cet effet anthropique, il est aussi important de noter que toutes ces stations sont localisées sur le bas Rhône. Ces stations sont donc proches de la mer Méditerranée, une barrière naturelle 
au flux d'espèces d'eau douce depuis des zones plus méridionales.

II est intéressant de noter que, malgré une augmentation générale des proportions d'espèces thermophiles sur le long terme, ces proportions tendent à diminuer avec la température à l'échelle inter-annuelle dans les stations localisées le long des ouvrages hydroélectriques. II existe une relation significativement négative entre la température moyenne et le débit moyen de la période de reproduction mesurés à proximité des stations (Daufresne \& Boët, 2007). Or, la plupart des espèces thermophiles présentes dans ces stations sont phyto-lithophiles (brème bordelière, tanche, gardon, poisson chat) ou lithophiles (brème) et déposent leurs œufs en bordure de rivière (Mann, 1995). Le recrutement de ces espèces est donc fortement influencé par l'environnement et en particulier par les risques d'assèchement (Mann, 1995). Malgré une augmentation globale de leurs proportions sur le long terme, le recrutement de ces espèces peut donc être ponctuellement réduit par des températures élevées et des débits faibles. De manière générale, les effets contradictoires d'une variable environnementale sur les abondances d'une espèce à différentes échelles temporelles ne sont pas surprenants. De fortes températures peuvent par exemple ponctuellement décroître le recrutement mais accroître la survie des juvéniles et des adultes, maintenant ainsi un taux de croissance positif de la population.

\section{- Aspects globaux}

II est primordial de bien distinguer l'effet des pressions non climatiques sur les dérives temporelles de la structure des communautés de leurs effets sur les structures en elles-mêmes. Si les aménagements hydroélectriques ont un effet globalement modéré sur les dérives temporelles, leurs effets sur les structures des communautés piscicoles ont très largement été démontrés (voir par ex : Bravard, 1987; Petts et al., 1989; Fruget, 1992; Kinsolving \& Bain, 1993). De même, si nous n'avons pas mis en évidence d'effets clairs des CNPE sur les dérives temporelles, il est vraisemblable qu'ils influencent la structure des communautés tout au moins dans la zone proche du rejet. En effet, les proportions des espèces principales ne sont significativement pas les mêmes en amont et en aval de 4 des 7 CNPE étudiés. L'analyse de la structure de la communauté à Bugey a également mis en évidence un effet du CNPE sur les espèces plus marginales, avec des proportions d'espèces thermophiles plus importantes et sténothermes d'eau froide moins importantes dans les stations soumises à l'influence du panache thermique. Une analyse plus approfondie de l'influence des CNPE sur l'ensemble des espèces présentes sur les différents sites devra confirmer ou infirmer la généralité de ce résultat. Dans ce sens, il apparaît aujourd'hui que le développement de nos connaissances sur l'impact des CNPE passe (i) par une description précise des conditions locales d'habitat physique (ex : hauteur d'eau, vitesse, substrat) et thermique (modélisation du panache thermique à l'aval des CNPE) et (ii) par une description de la variabilité locale (c'està-dire dans les stations soumises vs. non soumises à l'influence des CNPE) de l'écologie des espèces. En effet, il apparait par exemple probable que les différences de structure des communautés de poissons en amont et 
aval du CNPE Belleville soient, au moins pour partie, liées à des différences d'habitat physique (Lécureuil, com. pers.). Sans description précise de cet habitat la quantification de cet effet est toutefois aujourd'hui encore impossible.

\section{CONCLUSION}

Nous avons pu mettre en évidence des modifications importantes des abondances totales de poissons et de la structure et la diversité des communautés piscicoles en réponse au réchauffement des eaux. La plupart de ces dérives ne semblent que peu influencées par les aménagements hydroélectriques et les CNPE. Nous avons cependant pu mettre en évidence des effets de ces pressions anthropiques non climatiques sur la structure des communautés. Dans ce sens, nous ne pouvons pas exclure que ces différences ne conduiront pas, dans l'avenir, à des réponses différentes des communautés dans les différents sites. Quoiqu'il en soit, ces résultats soulignent qu'il est primordial de prendre en compte les effets du changement climatique en écologie des communautés, même lorsque l'on étudie des sites perturbés.

Enfin, cette étude souligne l'importance cruciale des suivis long terme en écologie dans le contexte du réchauffement global.

\section{REMERCIEMENTS}

Nous remercions EDF de nous avoir fourni les données et toutes les personnes qui ont collecté, saisi et corrigé ces données. La plupart des travaux présentés ont été réalisés et financés dans le cadre d'un accord cadre Cemagref-EDF.

\section{RÉFÉRENCES BIBLIOGRAPHIQUES}

Begon M., Harper J.L. \& Townsend C.R., 1990. Ecology : individuals, populations and community. Oxford : Blackwell Scientific Publications. 945 pp.

Bravard J.P., 1987. Le Rhône, du Léman à Lyon. Lyon : La Manufacture. $451 \mathrm{pp}$.

Bruslé J. \& Quignard J.P., 2001. Biologie des poissons d'eau douce européens. Paris : Éditions TEC\&DOC. 625 pp.

Cattanéo F., Carrel G., Lamouroux N. \& Breil P., 2001. Relationship between hydrology and cyprinid reproductive success in the lower Rhône at Montélimar, France. Arch. Hydrobiol. 151: 427-450.

Chatfield C., 1989. The Analysis of the Time Series : An Introduction. London : Chapman \& Hall. 241 pp.

Connell J.H., 1978. Diversity in tropical rain forests and coral reefs. Science 199 : 1302-1310.

Daufresne M. \& Boët P., 2007. Climate change impacts on structure and diversity of fish communities in rivers. Glob. Change Biol. 13 : 2467-2478.

Daufresne M., Roger M.C., Capra H. \& Lamouroux N., 2004. Long-term changes within the invertebrate and fish communities of the Upper Rhone River : effects of climatic factors. Glob. Change Biol. 10 : 124-140.

Daufresne M., Souchon Y., Villeneuve B. \& Capra H., 2005. Influence du rejet thermique de la centrale nucléaire de $\mathrm{Bu}$ gey sur la communauté de poissons du Rhône dans un contexte de réchauffement climatique. EDF.

Dembski S., Masson G., Monnier D., Wagner P. \& Phan J.C., 2006. Consequences of elevated temperatures on 
life-history traits of an introduced fish, pumpkinseed Lepomis gibbosus. J. Fish Biol. 69 : 331-346.

Elliott J.M., 1981. Some aspects of thermal stress on freshwater teleosts. In Stress and fish. Pickering A.D. (Ed.) : 36.

Fruget J.F., 1992. Ecology of the Lower Rhone after 200 Years of Human Influence - a Review. Regul. Rivers-Res. Manage. 7 : 233-246.

Grenouillet G., Hugueny B., Carrel G., Olivier J.M. \& Pont D., 2001. Largescale synchrony and inter-annual variability in roach recruitment in the Rhône River : the relative role of climatic factors and density-dependent processes. Freshw. Biol. 46 : 11-26.

Gurevitch J. \& Hedges V., 1993. Metaanalysis : combining the results of independant experiments. In Design and analysis of ecological experiments. Scheiner M. \& Gurevitch J. (Eds.) : 378-398.

Hamed K. \& Rao A., 1998. A modified Mann-Kendall trend test for autocorrelated data. Journal of Hydrology 204 : 182-196.

Hillebrand H., 2004. On the generality of the latitudinal diversity gradient. Am. Nat. 163 : 192-211.

Hughes L., 2000. Biological consequences of global warming : is the signal already apparent? Trends Ecol. Evol. $15: 56-61$.

Hutchinson G.E., 1961. The paradox of plankton. American Naturalist 95 : 137-145.

IPCC, 2001. Climate change 2001: the scientific basis. Cambridge : Cambridge University Press. 944 pp.

Keith P. \& Allardi J., 2001. Atlas des poissons d'eau douce de France. Paris : Patrimoines Naturels.

Kendall, 1955. Rank correlation methods. London : Griffin, $196 \mathrm{p}$.

Kinsolving A.D. \& Bain M.B., 1993. Fish assemblage recovery along a riverine disturbance gradient. Ecol. Appl. 3 : 531-544.

Knouft J.H., 2004. Latitudinal variation in the shape of the species body size distribution : an analysis using freshwater fishes. Oecologia 139 : 408-417.

Kraiem M.M., 1979. Écologie du barbeau fluviatile Barbus barbus (L.1758) [Poissons, cyprinidae] dans le Haut-Rhône français. Thèse de troisième cycle, Université Claude Bernard-Lyon1. Lyon : 150 pp.

Küttel S., Peter A. \& Wüest A., 2002. Temperaturpräferenzen und -limiten von Fischarten Schweizerischer Fliessgewässer. EAWAG. Kastanienbaum : $41 \mathrm{pp}$.

Mann R.H.K., 1995. Natural factors influencing recruitment success in coarse fish population. In The ecologicla basis for river management. Harper D.M. \& Ferguson A.J.D. (Eds.) : 339-348.

Parmesan C. \& Yohe G., 2003. A globally coherent fingerprint of climate change impacts across natural systems. Nature $421: 37-42$.

Petts G.E., Moeller H. \& Roux A.L., 1989. Historical change of large alluvial rivers : western Europe. Chichester : John Wiley \& sons.

Philippart J.C. \& Vranken M., 1983. Atlas des poissons de Wallonie. Cahiers Ethol. Appl. 3 : 1-395.

Proteau J.P., Hilge V. \& Linhart O., 1996. Present state and prospects of the aquaculture of catfishes (Siluroidei) in Europe. Aquat. Living Resour. 9 : 229235.

Pyper B.J. \& Peterman R.M., 1998. Comparison of methods to account for autocorrelation in correlation analyses of fish data. Can. J. Fish. Aquat. Sci. 55 : 2127-2140.

R Development Core Team, 2008. R: A language and environment for statistical computing. R Foundation for Statistical Computing, Vienna, Austria. ISBN 
3-900051-07-0, http://www.R-project. org.

Root T.L., Price J.T., Hall K.R., Schneider S.H., Rosenzweig C. \& Pounds J.A., 2003. Fingerprints of global warming on wild animals and plants. Nature 421: 57-60.

Scott W.B. \& Crossman E.J. (Eds.), 1973. Freshwater fishes of Canada, Bull. Fish. Res. Board Can. Ottawa : 966 pp.

Sen P.K., 1968. Estimates of regression coefficient based on Kendall's tau. 63 : 1379-1389.

Sokal R.R. \& Rohlf J., 1995. Biometry : the principles and practice of statistics in Biological research. New York : Freeman. $887 \mathrm{pp}$.

Walker M.D., Wahren C.H., Hollister R.D., Henry G.H.R., Ahlquist L.E., Alatalo J.M., Bret-Harte M.S., Calef M.P., Callaghan T.V., Carroll A.B.,
Epstein H.E., Jonsdottir I.S., Klein J.A., Magnusson B., Molau U., Oberbauer S.F., Rewa S.P., Robinson C.H., Shaver G.R., Suding K.N., Thompson C.C., Tolvanen A., Totland O., Turner P.L., Tweedie C.E., Webber P.J. \& Wookey P.A., 2006. Plant community responses to experimental warming across the tundra biome. Proc. Natl. Acad. Sci. U. S. A. 103 : 1342-1346.

Walther G.R., Post E., Convey P., Menzel A., Parmesan C., Beebee T.J.C., Fromentin J.M., Hoegh-Guldberg O. \& Bairlein F., 2002. Ecological responses to recent climate change. Nature 416 : 389-395.

Wilson J.B., 1990. Mechanisms of Species Coexistence - 12 Explanations for Hutchinson Paradox of the Plankton Evidence from New-Zealand PlantCommunities. N. Z. J. Mar. Freshw. Res. 13:17-42. 